\title{
Adaptive Routenbeschreibungen für Navigationssysteme
}

\section{Adaptive Presentation of Itineraries in Navigation Systems}

Routenpräsentation_Navigationssysteme_Adaption

\begin{abstract}
Zusammenfassung. In diesem Beitrag stellen wir ein Verfahren zur adaptiven Präsentation für Navigationsgeräte vor, welche auf semantischen Modelle nasiert. Dabei reichern wir herkömmliche Routenbeschreibungen mit semantischen Informationen an und präsentieren dem Fahrer lediglich diejenigen Routenanweisungen, an denen er wirklich interessiert ist und blenden überflüssige Anweisungen aus.
\end{abstract}

\section{Einleitung}

Aktuellen Erhebungen (Institut für Demoskopie Allensbach, 2010) zufolge hat sich allein in Deutschland der Anteil vorinstallierter Navigationssysteme in Neufahrzeugen innerhalb der letzten sechs Jahre von weniger als $6 \%$ auf $18 \%$ erhöht. Der Anteil mobiler Navigationsgeräte stieg im gleichen Zeitraum sogar von 1\% auf fast 31\%. Wenngleich der wirtschaftliche Erfolg als Bestätigung für die Beliebtheit solcher Geräte angesehen werden kann, zeigen neuere Forschungsergebnisse, dass die Gebrauchstauglichkeit durch die wachsende Funktionsvielfalt zunehmend leidet (Hipp et al., 2010).

Leistungsfähigere Endgeräte erlauben immer bessere Ausgabemöglichkeiten, wie Text-to-Speech oder die realistischere Umgebungsdarstellung. Andererseits birgt das die Gefahr einer Überforderung der Anwender. Die zunehmende Informationsflut, der wachsende Detailgrad in der Darstellung und die vielfältigen Interaktionsmöglichkeiten sind besonders bei kleinen Displays problematisch. Der Anwender muss sich stärker auf die Interaktion mit dem Navigationsgerät konzentrieren, obwohl seine primäre Aufgabe darin besteht, ein Fahrzeug sicher im Straßenverkehr zu bewegen. Detaillierte Fahranweisungen oder die Präsentation von Zusatzangeboten, können den Fahrer stören und ablenken. Insbesondere in diesem Bereich gibt es noch Spielraum für weitere Verbesserungen. Anwender wünschen sich durchaus eine einfachere Interaktion mit einem GPS-Gerät, da sie diese während der Fahrt als nicht sicher einschätzen (Al Mahmud et al. 2009).

Derzeitige Lösungen streben vornehmlich eine Routenoptimierung an, ohne das persönliche das Vorwissen, die Erfahrung und die individuellen Präferenzen des Fahrers zu berücksichtigen und sind daher nicht personalisiert. Üblicherweise ist die Darstellung der Route zu jeder Zeit des Navigationsprozesses sehr detailliert - selbst wenn der Fahrer Teile der Strecke sehr gut kennt. Die Einbeziehung persönlicher Informationen könnte die Routendarstellung erheblich verbessern. Bereits in einer früheren Untersuchung über die menschliche Erstellung und Kommunikation von Routenbeschreibungen konnte gezeigt werden, dass die Erwähnung einzelner Segmente einer Route in bekannten Umgebungen weniger relevant ist (Lovelace et al., 1999). Eine aktuellere Studie zu kollaborativen Navigationsstrategien lieferte ein ähnliches Ergebnis. Dabei konnte bestätigt werden, dass sich die Mehrzahl der Probanden nur in ihnen unbekannten Bereichen explizit auf den Wegfindungsprozess konzentrierten, während sie sich in eher bekannten Regionen zeitgleich auch anderen Themen widmen konnten (Forlizzi et al., 2010). Die personalisierte Darstellung der Routenbeschreibung in Abhängigkeit von individuellen Anforderungen und Vorlieben wurde auch im Rahmen der von Mahmud et al. (2009) durchgeführten Nutzerstudie als eine der Hauptanforderungen an moderne Navigationssysteme gesehen. Kennt sich der Benutzer in einem bestimmten Bereich gut aus, sollten Fahranweisungen eher kurz und abstrakt sein. Fährt er dagegen 
durch unbekanntes Gebiet, erfordert dies eine detailliertere Präsentation.

In diesem Beitrag stellen wir ein Konzept zur Anpassung der Routenpräsentation an die Erfahrungen und Vorlieben des Fahrers vor. Zu diesem Zweck benutzen wir semantisch angereicherte Modelle der Routenbeschreibung. Im Ergebnis sieht und hört der Benutzer anstelle jedes einzelnen Details nur die für inn notwendigen und hilfreichen Informationen, die Präsentation wird also automatisch an ihn angepasst. Neben der automatisierten Adaption hat der Benutzer dabei stets die Möglichkeit, den Detailgrad der Darstellung manuell zu beeinflussen.

\section{Beschreibung von Verkehrsrouten}

Routenbeschreibungen kommunizieren die Fahranweisungen entlang einer Strecke vom Start- zum Zielort. Diese "route directions" können sowohl von Menschen als auch computergestützten Systemen generiert werden und lassen sich als aufgabenorientierte Spezifikationen verstehen, die zur Erreichung eines Zielorts erforderlich sind (Richter et al., 2008). Ihr Hauptzweck ist die Unterstützung bei der Bestimmung der Vorgehensweise an den jeweiligen Entscheidungspunkten („decision points “) einer Routenbeschreibung.

\section{Formen der Routenbeschreibung}

Die entsprechenden Anweisungen werden dabei dem Fahrer an den Entscheidungspunkten präsentiert. Dieser muss dann agieren, etwa auf eine Autobahn auffahren oder an einer Kreuzung abbiegen. Die zugehörigen Anweisungen, die den Fahrer von einem Entscheidungspunkt zum nächsten führen und dabei sukzessive über jeden notwendigen Richtungswechsel informieren, werden auch als „turn-by-turn directions" bezeichnet.

"Turn-by-turn directions" beantworten also die Frage, „wie” ein Zielort erreicht werden kann. Das ist insbesondere dann hilfreich, wenn beim Fahrer keinerlei Kenntnis über die Route vorliegt. Dies entspricht allerdings in der Regel nicht der Realität, da Routen zumeist in bekanntem Terrain (Wohnort, Arbeitsplatz) beginnen oder enden.

Reine „turn-by-turn“-Anweisungen entsprechen zudem nicht der intuitiven menschlichen Instruktionsweise. Persönlich kommunizierte Wegbeschreibungen verzichten in der Regel auf eine Vielzahl von Details. Stattdessen wird zunächst räumliches Wissen über die Umgebung aktiviert, relevante Informationen identifiziert und strukturiert und anschließend in verbalisierter Form kommuniziert (Lovelace et al., 1999). Dabei werden häufig mehrere aufeinanderfolgende Entscheidungspunkte zu einer übergeordneten Beschreibung zusammengefasst. Klippel et al. (2003) bezeichnen dies als „spatial chunking".

Als Beispiel dafür dient die Anweisung, "an der dritten Ampel rechts" zu fahren, wobei mindestens zwei Ampeln inkludiert aber nicht explizit genannt werden. Dabei handelt es sich um sogenanntes numerisches Chunking, also das Zusammenfassen einer gewissen Anzahl von Objekten gleichen Typs. Eine alternative Vorgehensweise bietet das Chunking auf Basis von Landmarks, also markanten Punkten entlang einer Route, etwa ein Turm oder ein Autobahnanschluss. Dabei würde eine Anweisung etwa lauten: „Fahren Sie zur Autobahn” (vgl. Klippel et al., 2009).

Eine weitere Vorgehensweise ist, das Fahrtziel anhand seiner Umgebung näher zu beschreiben. Das kann beispielsweise für einen Taxifahrer hilfreich sein, der sich zwar in seiner Stadt gut zurecht findet, jedoch nicht jede einzelne Straße kennt. Wird inm beschrieben, wo sich die Zielstraße befindet, etwa "hinter dem Theater am Marientor", welches er gut kennt, kann er den Weg dorthin leichter finden. Dieser Ansatz wird als "destination description" (Tomko, 2007) bezeichnet. Personen, die mit der Umgebung vertraut sind, können also Beschreibungen von Routen durch Referenzen auf innen bekannte Objekte liefern. Bei dieser Art der Beschreibung wird gewissermaßen angegeben, "wohin" als nächstes gefahren werden soll.

"Destination descriptions" und das in menschlichen Routenbeschreibungen häufig verwendete "spatial chunking" haben hier ihre Begründung. Es existiert demnach eine direkte Abhängigkeit zwischen der Umgebungskenntnis und dem Detailgrad humaner Routenbeschreibungen - ein Umstand, der bei computergestützter Generierung bislang nicht ausreichend berücksichtigt wird, obwohl diese Abstraktionsmöglichkeit zur Handhabung der reichhaltigen Umgebungsinformationen sowie als kognitiver Prozess ein essentieller Teil der menschlichen Informationsverarbeitung ist (Klippel et al., 2009). Stattdessen präsentieren aktuelle Navigationslösungen konsequent die Anweisungen für jeden einzelnen Entscheidungspunkt. Dadurch steigt die kognitive Belastung des Fahrers unnötig an, was im Extremfall zu gefährlichen Situationen führen kann (Pettitt et al., 2005).

\section{Existierende Prototypen}

Mit „MyRoute” stellen Patel et al. (2006) einen Ansatz zur Routenpersonalisierung vor, der eine Streckenbe-schreibung in zwei Schritten komprimiert. Im ersten Schritt werden Landmarks, die der Benutzer bereits kennt, und die Routeninformationen in ein personalisiertes Profil zusammengefasst. Die bekannten Landmarks sowie ihre Verbindung untereinander müssen zuvor in einem separaten Prozess vom Benutzer aufwendig eingepflegt werden. Im zweiten Schritt nutzt die Anwendung das erstellte Profil zur Vereinfachung des Weges, indem als bekannt gekennzeichnete Landmarks in Routennähe als Zwischenziele fungieren, die der Benutzer auch ohne Navigationsunterstützung erreichen kann. Die Anweisungen werden anschließend an diese Landmarks angepasst, womit das oben beschriebene Konzept des Landmarkbasierten Chunkings nach Klippel et al. (2009) zur Anwendung kommt. Trotz Verwendung einer Kostenfunktion kann sich bei dieser Lösung im ungünstigen Fall auch eine wesentlich längere als die ursprünglich berechnete Strecke ergeben. Die Autoren argumentieren, dass der Anwender dafür entlang einer inm vertrauten Route geleitet wird und die Reduzierung der Routenkomplexität weniger Ablenkungen verursacht, wodurch die Fahrt stressfreier und damit auch sicherer verläuft. Der Ansatz fokussiert weniger auf die Präsentation als vielmehr auf die algorithmische Umsetzung.

Einen anderen Vorschlag, adaptive Routenanweisungen basierend auf einer Kombination aus "Turn-by-turn Directions" und "Destination Descriptions" zu erstellen, präsentieren Richter et al. (2008). Die Arbeit fokussiert dabei eher die Problem-Formalisierung sowie ihre 
algorithmische Umsetzung. Ihr Ziel ist, adaptive Routenanweisungen automatisch und generisch zu erstellen, ohne dabei das individuelle räumliche Vorwissen eines Benutzers einbeziehen zu müssen. Diese Art der Routenpräsentation erfordert stattdessen eine (u.U. permanente) Interaktion des Anwenders während der Fahrt. Indem initial für alle Benutzer die jeweilige Umgebung als bekannt angenommen wird, soll die Problematik lernender Systeme umgangen werden. Die Fahranweisungen erfolgen in diesem Zustand als "Destination Descriptions", wobei bekannte Landmarks als Zwischenziele fungieren. Benötigt der Anwender detailliertere Informationen, muss er diese über einen Dialog anfordern und erhält dann "Turn-by-turn Directions", bis der nächste Landmark erreicht wird. Dann schaltet die Anwendung wieder in den ursprünglichen Modus. Diese Vorgehensweise erfordert vom Benutzer eine ständige Interaktion mit dem Endgerät während der Fahrt, wenn er sich in einem Bereich nicht auskennt. Dies birgt die Gefahr häufiger Ablenkung und ist daher aus sicherheitstechnischer Perspektive kritisch zu sehen

\section{Ein Ansatz zur perso- nalisierten optimierten Routendarstellung}

Hat ein Benutzer ausreichende Kenntnisse über die Umgebung eines bestimmten Bereiches, genügen wenige Anweisungen, beispielsweise die nächstgelegene Autobahn und anschließende Fahrtrichtung. In Bereichen, in denen sich ein Benutzer dagegen weniger auskennt oder die ihm gar nicht vertraut sind, ist eine ausführliche Zielführung gänzlich ohne Abstraktion die geeignetere Wahl.

Der in diesem Artikel vorgestellte Ansatz verbindet die im letzten Abschnitt vorgestellten Ideen. Eine optimale Routendarstellung sollte in bekannten Gebieten eher gemäß des Prinzips der "destination descriptions" vorgehen („fahre Richtung Köln"), in unbekanntem Terrain jedoch traditionelle "turn-by-turn"-Direktiven verwenden. Um zwischen diesen Extremen überzuleiten, werden in Anlehnung an das „chunking"-Konzept Anweisungen mal mehr, mal weniger stark gebündelt präsentiert.
Grundidee ist, für eine Route jeweils mehrere unterschiedliche Darstellungen in abgestufter Granularität zu erstellen. Während der Fahrt wird die Präsentation dann an den Fahrer adaptiert, indem situativ zwischen den Ebenen gewechselt wird. So kann flexibel zwischen detailreichen und -armen Ebenen umgeschaltet werden und auf das Nutzerwissen und dessen Präferenzen eingegangen werden. Der Fahrer erhält eine auf seine Kenntnis angepasste Präsentation während gleichzeitig die Notwendigkeit der Interaktion während der Fahrt auf das Nötigste reduziert bleibt.

Im Folgenden werden nun die zwei grundlegenden Bestandteile dieser Lösung beschrieben: Zunächst wird mit semantischen Routenmodelle die Grundlage der Abstraktionsebenen beschrieben. Diese Modelle werden für jede Route ad hoc aus einfachen XML-Beschreibungen von Google Maps gewonnen, indem die dort erhaltenen Informationen durch Geo-Services angereichert werden und in RDF-Markup überführt werden. Um die Darstellung zu personalisieren, werden zudem Informationen über den Nutzer benötigt, welche in möglichst unaufdringlicher Weise gesammelt und genutzt werden sollten. Die Anwendung kann dann basierend auf diesen Daten einen Detailgrad für einzelne Routenabschnitte vorschlagen, wobei der Anwender jederzeit auch in einen höheren Abstraktionsgrad wechseln oder auch mehr Details anfordern kann. Zudem soll dem Nutzer jederzeit die Möglichkeit gegeben werden, das vom System adaptierte Verhalten zu korrigieren.

\section{Semantische Routen- modelle als Basis für personalisierte Präsen- tation}

Eine Route besteht aus einer Menge von Wegpunkten, die einzelne Positionen zwischen Start und Ziel repräsentieren. Die Idee ist nun, diese Wegpunkte se-

1 Das Resource Description Framework (RDF) ist eine Familie von Standards zur semantischen Beschreibung von Objekten sowie ihren Beziehungen untereinander und ist etablierter Bestandteil vieler Semantic Web Anwendungen. mantisch anzureichern, um diese semantischen Informationen für die spätere Filterung verwenden zu können. Dazu müssen alle Informationen einbezogen werden, die für die Entscheidung, ob ein bestimmter Teil der Route sehr detailliert, eher abstrakt oder überhaupt nicht dargestellt werden soll, hilfreich sein können.

Wir schlagen dazu ein Ebenenmodell vor, bei dem jede Ebene einen Detailgrad der Routenbeschreibung abbildet. Die unterste Ebene beinhaltet die ursprüngliche Routenbeschreibung mit allen Details der Strecke, wie es von aktuellen Navigationsgeräten bekannt ist. Alle darüber liegenden Ebenen zeigen in Abhängigkeit vom Abstraktionsgrad nur noch bestimmte Teile der Routenbeschreibung und die entsprechenden Fahranweisungen.

Um dieses Ziel zu erreichen, transformieren wir die ursprüngliche Routenbeschreibung in ein semantisches Modell, welches uns erlaubt, jeden Wegpunkt anhand seiner Eigenschaften zu charakterisieren. Zusätzlich zu den allgemeinen Informationen der Strecke, wie Ortskoordinaten, Straßenname und Fahranweisungen, beinhaltet die angereicherte semantische Beschreibung weitere Informationen, wie die Klassifikation jedes Wegpunktes auf Basis seines Typs oder der geografischen Gegebenheiten. So kann ein Ort beispielsweise als Dorf, Stadt oder Region klassifiziert werden und die Verbindung zwischen zwei Orten kann eine Straße, eine Bundesstraße oder eine Autobahn sein. Diese hierarchische Unterscheidung ermöglicht die spätere Filterung zur Bestimmung der verschiedenen Abstraktionsebenen. Für diese Transformation eignet sich grundsätzlich jede beliebige Routenbeschreibung, wie sie von webbasierten Diensten, wie Google Maps, bereitgestellt wird. Zur Anreicherung semantischer Routenbeschreibungen mit zusätzlichen Informationen können Dienste, wie LinkedGeoData.org, OpenStreetMap.org, GeoNames.org oder TopoCoding.com verwendet werden. Derartige Dienste stellen ein umfassendes Hintergrundwissen über die räumlichen Bedingungen, Struktur und Landschaften entlang einer Strecke, zur Verfügung

Ein Navigationsgerät kann so beispielsweise ausschließlich prominente Wegpunkte präsentieren, wie Bundesstraßen (sofern der Fahrer grundlegende Kenntnisse von der Umgebung hat). In 


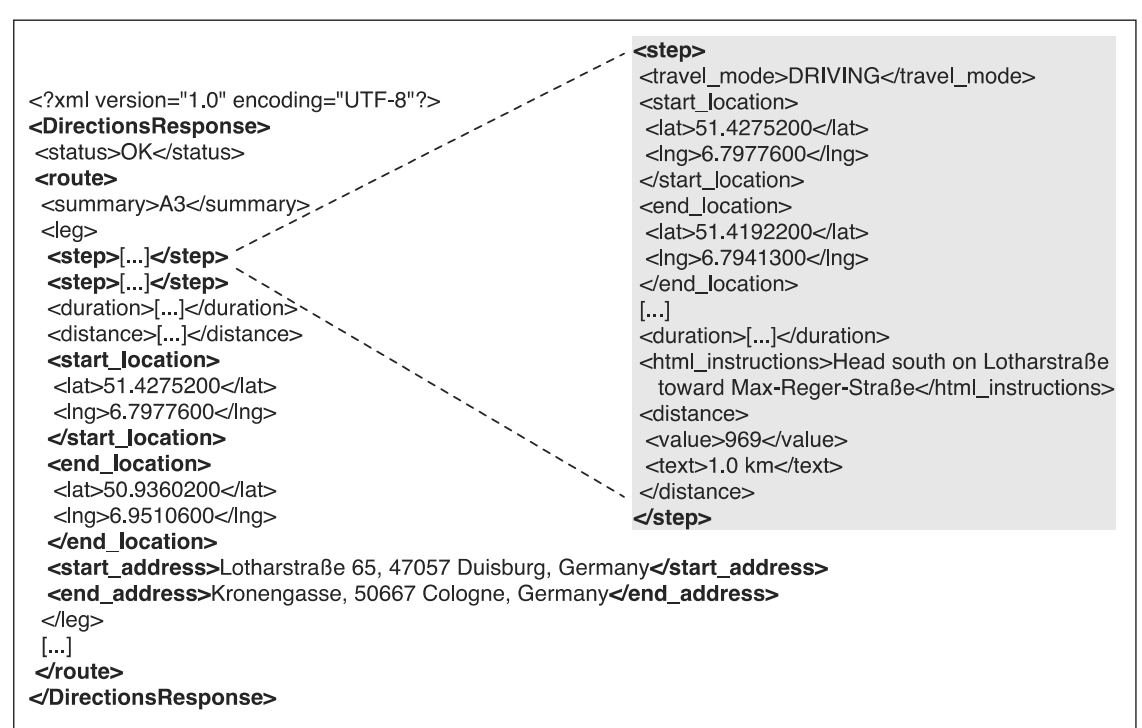

Bild 1: Typischer Aufbau einer XML-basierten Routenbeschreibung, wie sie von Google Maps exportiert werden kann. Die einzelnen Wegpunkte werden dabei als "steps" definiert und enthalten bereits wichtige Informationen wie GPS-Positionsangaben, die im Folgenden zur Weiterverarbeitung genutzt werden können.

diesem Fall könnte eine Fahranweisung vereinfacht lauten: „Fahren Sie zur Autobahn 3", während andere Benutzer durch eine Reihe detaillierter Anweisungen zu dieser Autobahn geführt würden.

Grundlage der dargestellten Präsentationsstrategie sind semantische Modelle der entsprechenden Routen. Verglichen mit herkömmlichen Wegbeschreibungen, bietet die Verwendung semantischer Modelle insbesondere zwei Vorteile:

- Standardisierung: Sofern Informationen von verschiedenen Quellen stammen, jede mit anderem Format und Spezifikation, wird ein Standard benötigt, die diese unterschiedlichen Formate kapselt. Semantische Modelle sind flexibel genug, um Informationen aus heterogenen Quellen zu vereinen und in einheitlicher Weise verfügbar zu machen. So können sie mit Hilfe etablierter Anfragesprachen wie SPARQL weiterverarbeitet werden.

- Erweiterbarkeit: Die oben beschriebenen Eigenschaften semantischer Modelle erlauben ebenso die Integration neuer Informationen, unabhängig von ihrem Format. Diese Informationen wiederum legen den Grundstein für künftige Dienste, die über die klassische Routenführung hinausgehen. Empfehlungssysteme, die semantische Daten einbeziehen, können beispielsweise Tankstellen mit attraktiven
Sonderangeboten oder beliebte Restaurants entlang der Strecke finden und empfehlen. Ideen für derartige Mehrwertdienste wurden bereits von den Autoren vorgestellt (Münter et al., 2010).

\section{Semantifizierung von Wegbeschreibungen}

Da ausführliche semantische Informationen zu Streckenbeschreibungen nicht in geeignetem Maße vorliegen, beruht der vorgestellte Ansatz auf einer ad-hocUmwandlung traditioneller Routenbeschreibungen in semantische Modelle. Im weiteren Verlauf wird dieser Prozess als "Semantifizierung" bezeichnet, bei der die folgenden Schritte durchgeführt werden.
Ausgangsbasis ist eine XML-basierte Beschreibung der Route, welche über Google Maps bezogen werden kann (Bild 1).

Die Informationen zu den einzelnen Wegpunkten ( "steps") werden daraufhin mit Hilfe externer Geo-Services wie OpenStreetMap.org oder GeoNames. org auf Basis der geografischen Koordinaten um weitere Fakten angereichert: In welcher Stadt befindet sich der aktuelle Wegpunkt, welcher Straßentyp liegt vor, etc. Zusammen mit diesen Informationen können die Wegpunkte nun in semantische Statements transformiert werden (Bild 2). Intern erfolgt die Kodierung hierbei nach der im Semantic Web etablierten Auszeichnungssprache RDF.

\section{Filterung der Wegpunkte}

Derart semantisch angereichert, können die einzelnen Wegpunkte nun nach unterschiedlichen Anforderungen gefiltert werden. Hierzu wird die semantische Abfragesprache SPARQL genutzt. Bild 3 zeigt eine solche SPARQL-Abfrage, die die Wegpunkte herausfiltert, die Städte mit mehr als 100.000 Einwohnern repräsentieren. So können Anweisungen wie "Fahre in Richtung Düsseldorf" realisiert werden, um bei relativ bekanntem Terrain keine unnötig detaillierten Instruktionen anzuzeigen.

Derartige SPARQL-Anfragen können hochgradig flexibel definiert werden, um je nach gewünschten Detailgrad bestimmte Wegpunkte ein- oder auszublenden.

\section{Generierung von Abstraktionsebenen}

Ein wesentlicher Zwischenschritt auf dem Weg zu einer personalisierten Routen-

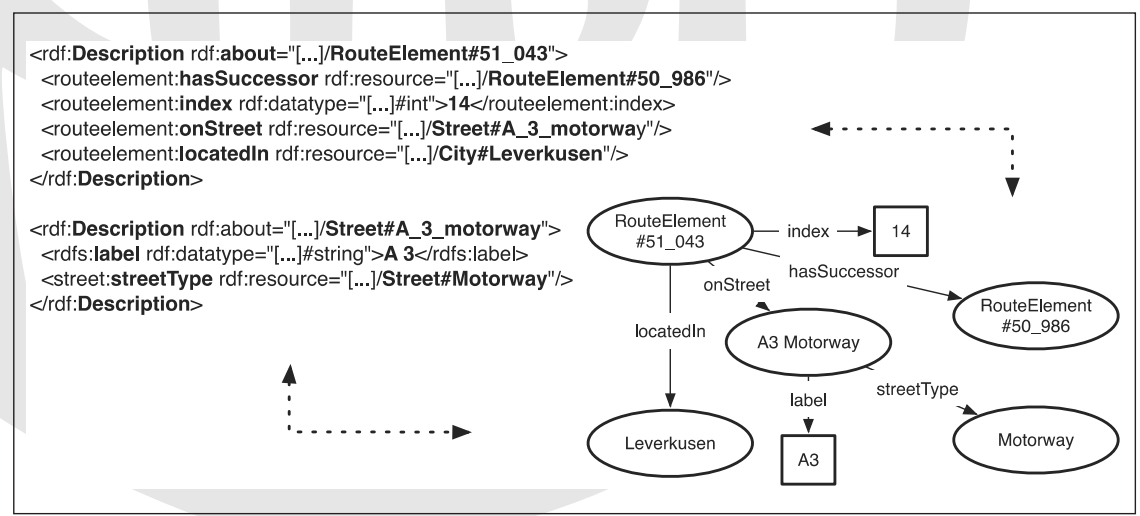

Bild 2: Modell eines semantifizierten Wegpunktes, welches die Informationen über den betreffenden Streckenabschnitt enthält. Links die Kodierung als RDF-Aussagen, rechts als Schaubild. 


\section{SELECT DISTINCT ?city WHERE \{ \\ \{ \\ ?routeElement routeelement:locatedln ?city . \\ ?city rdfs:label ?cityName . \\ ?city city:population ?cityPopulation . \\ FILTER (?cityPopulation > 100000) \\ \} \\ ?routeElement routeelement:index ?index . \} ORDER BY (?index)}

Bild 3: SPARQL-Abfrage, welche nur diejenigen Wegpunkte anzeigt, welche größere Städte repräsentieren

präsentation ist die Generierung eines Ebenenodells aus der semantischen Routenbeschreibung (Bild 4). Die einzelnen Ebenen sollen den jeweiligen Abstraktionsgrad widerspiegeln. Die unterste Schicht beinhaltet dabei alle Wegpunkte, während der Detailgrad mit jeder höheren Ebene abnimmt

Aus der Ursprungsmenge aller Wegpunkte lassen sich die für eine Ebene relevanten Orte extrahieren. Dazu wird die SPARQL-Anfrage mit den entsprechenden Filterbedingungen versehen. Das können Attribute wie der Typ des Ortes oder auch die Tatsache sein, dass sich der Benutzer in diesem Bereich gut auskennt. Auf diese Weise lassen sich Wegpunkte in Abhängigkeit von der gewählten Abstraktionsebene darstellen oder verbergen. Jede Ebene kann auch als "Sicht auf die Route" betrachtet werden, die bestimmte Details zeigt oder verbirgt. Die Basisebene stellt dabei analog zu herkömmlichen Navigationsgeräten alle Details dar (reines "turn-by-turn"). Wird eine abstraktere Darstellung gewählt, werden weniger relevante Wegpunkte ausgeblendet und stattdessen nur noch für die jeweilige Ebene notwendige Wegpunkte präsentiert.

\section{Adaptive \\ Routenpräsentation}

Das vorgestellte Ebenenmodell erlaubt nun das Umschalten zwischen den Detailsichten, ähnlich dem Zoomen auf der Kartendarstellung. Im Sinne einer einfachen, unaufdringlichen und zufriedenstellenden Nutzung sollte einerseits eine automatische Adaption an den Nutzer stattfinden, die sich an seiner Ortskenntnis orientiert, andererseits sollte immer die Möglichkeit der manuellen Anpassung gegeben sein.

\section{Dialogbasierte Routenanpassung}

Eine einfache Form der Darstellungsanpassung kann durch Interaktion mit dem Fahrer erreicht werden. Dazu sollte der Benutzer unmittelbar nach Berechnung der Route inm bekannte Bereiche der Strecke in einem eigenen Dialog kennzeichnen können. Dieses Vorgehen ist ähnlich dem von Richter beschriebenen dialog-getriebenen Prozess.

Der wesentliche Unterschied im vorgestellten Ansatz ist, dass wir den Dialog zuerst verwenden, um die gesamte Routenführung auf den individuellen

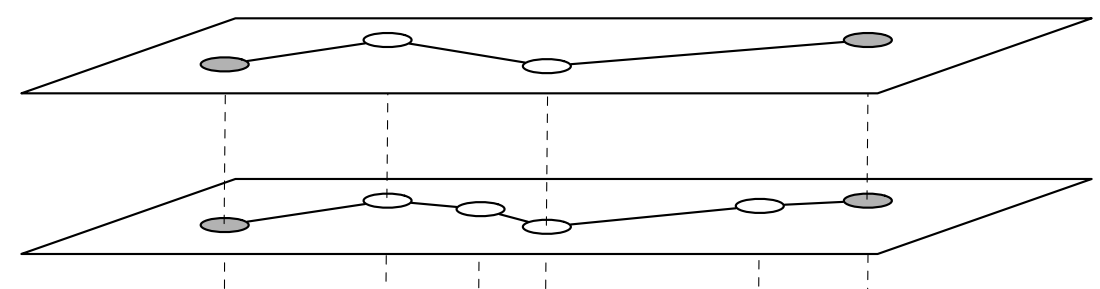

Bild 4: Verschiedene Granularitätsgrade einer Routenbeschreibung in Form von Abstraktionsebenen. Während die unterste Ebene alle Informationen enthält, werden die Wegpunkte auf höheren Ebenen immer stärker gefiltert bis ausschließlich die wichtigsten Punkte enthalten sind.
Bedarf des Benutzers abstimmen. Richter hingegen stellt standardmäßig abstrakte Anweisungen zur Verfügung und fordert Benutzerinteraktionen immer dann, wenn der Fahrer detailliertere Instruktionen benötigt. Diese Art der Interaktionsmöglichkeit stellen wir zusätzlich zur Verfügung. Der Benutzer kann mit Hilfe eines einfachen Interaktionsschrittes den Abstraktionsgrad zu jeder Zeit manuell setzen und justieren.

Diese Funktionalität ist zu jeder Zeit des Routenführungsprozesses verfügbar und soll dem Benutzer jederzeit ermöglichen, entsprechend seinen individuellen Bedürfnissen eine angemessene Darstellung der Routenbeschreibung zu erhalten. Die Möglichkeit, jederzeit mit dem System interagieren zu können, erhöht auch die Zufriedenheit des Anwenders und kann so auch die Akzeptanz eines automatisierten Prozesses fördern. Bild 5 zeigt ein solches Interaktions-Widget. Dabei stehen dem Anwender zwei Buttons zur Verfügung, mit denen er den Detailgrad der Darstellung beeinflussen kann. Ein Klick auf die Schaltfläche "More" bewirkt eine detailliertere Darstellung sowohl in der Ansicht als auch hinsichtlich der Fahranweisungen. Der "Less"-Button dagegen ermöglicht eine abstraktere Präsentation mit weniger Details.

\section{Benutzermodelle und automatische \\ Anpassung}

Zur Generierung reichhaltigerer Anpassungseffekte können dedizierte Nutzerprofile verwendet werden. Sie ermöglichen dem System, auf die individuellen Präferenzen und das Umgebungswissen des Anwenders zu reagieren, sofern dieser bestimmte Bereiche der Route als bekannt markiert. Es kann dann bei neu berechneten Routen einen Detailgrad empfehlen. Dazu wird ein Benutzermodell sukzessive mit Informationen angereichert, die sich aus der Interaktion über einen Dialog einerseits oder aus bereits befahrenen Bereichen andererseits ergeben. Bild 6 zeigt eine Karte, auf der exemplarisch das Umgebungswissen eines Benutzers abgebildet ist. Dabei sind die bekannten Bereiche dunkler dargestellt und weniger bekannte Bereiche heller.

Die Eingabe erfolgt über ein Webinterface, in welchem der Nutzer seine Kenntnis der Umgebung von Bundesländern, 


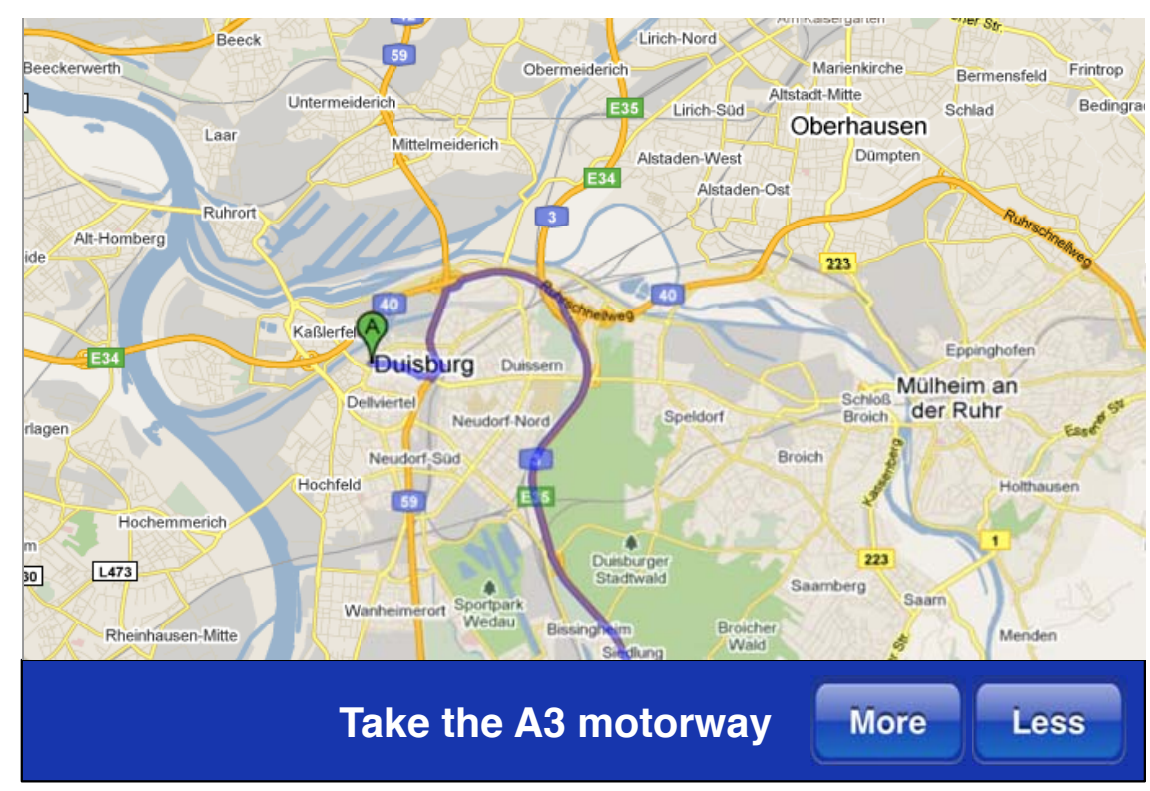

Bild 5: Ein Interaktions-Widget ermöglicht das manuelle Umschalten zwischen den Abstraktionsgraden.

über Städte und Postleitzahlbereichen abgestuft einstellen kann. StandardmäBig wird die gesamte Straßenlandschaft Deutschlands hierbei als unbekannt vorausgesetzt, was eine Präsentationsweise gemäß der von herkömmlichen Navigationssystemen zur Folge hätte: Turn-byturn-Anweisungen in jedem Abschnitt. Mit steigendem Bekanntheitsgrad steigt der Abstraktionsgrad der Beschreibungen bis hin zu Destination-based Anweisun- gen, die lediglich die Städte beinhalten („Fahren Sie Richtung Köln“).

\section{Erfahrungen}

Auf Basis der beschriebenen Konzepte ist im Rahmen des Projektes colognE-mobil der Prototyp eines intelligenten Mobilitätsassistenten entstanden. In einer ersten Version wurde dieser als Desktop-Simulator entworfen. Dieser benutzt Schnittstel-

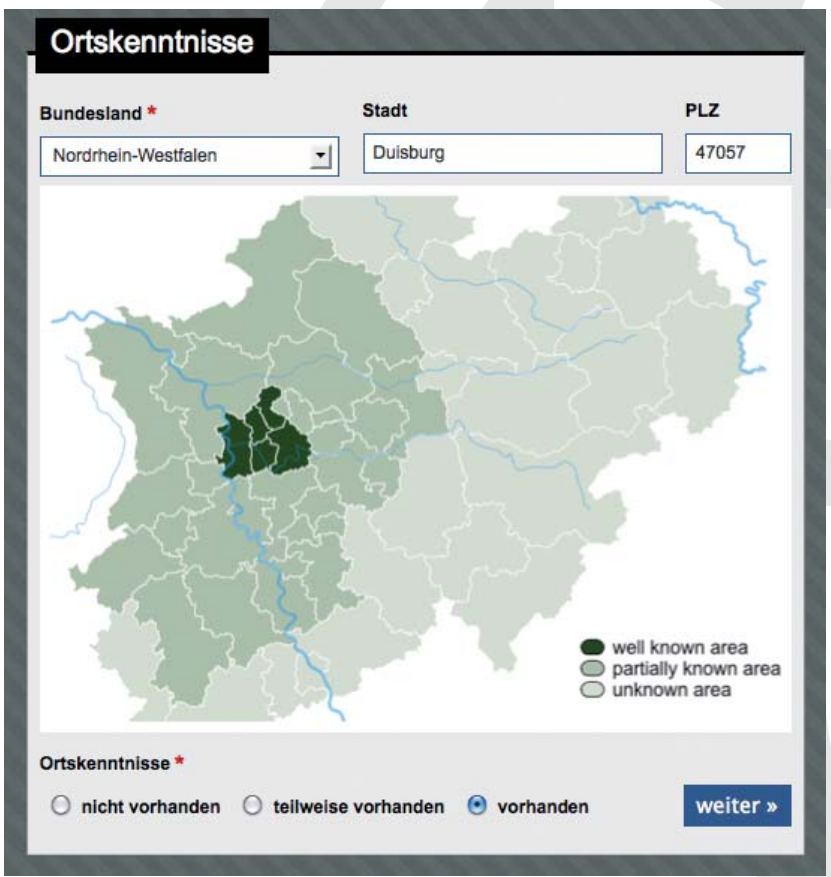

Bild 6: Ein Web-Interface erlaubt es dem Benutzer, seine Ortskenntnisse in das Benutzermodell der Anwendung zu übertragen. len öffentlich zugänglicher Webdienste, wie der Google API. Die Präsentation berechneter Routen erfolgt dabei nach folgendem Schema: zunächst werden dem Benutzer alle Details der Route angezeigt, um ihm einen Gesamtüberblick zu gewähren. Anschließend werden unter Berücksichtigung des persönlichen Nutzerprofils diejenigen Details ausgeblendet, die für den aktuellen Nutzer als überflüssig erachtet werden. Durch die positive Resonanz auf diesen ersten Prototypen wurde schließlich eine Umsetzung für das Apple iPad implementiert, welche die aktuellen GPS-Informationen des Nutzers berücksichtigen kann.

Erste Erfahrungen im Umgang mit dem Prototypen zeigten, dass Benutzer diese neue Art der Routendarstellung insbesondere in ihnen bekannten Bereichen als sehr angenehm und hilfreich empfunden haben im Vergleich zu herkömmlichen Routenanweisungen.

\section{Diskussion und Ausblick}

Der vorgestellte Ansatz baut auf bestehenden Ideen der Navigationsmodellierung auf und fügt eine interaktive, nutzeradaptive Komponente hinzu. In der prototypischen Implementierung ist eine Interaktion des Benutzers mit dem System zur Erstellung eines Benutzerprofils erforderlich. Diese erfolgt entweder mit Hilfe eines vorgeschalteten Dialogs, in dem der Anwender anhand der Beschreibung der berechneten Route ihm bekannte Wegpunkte als solche kennzeichnen kann, oder unter Verwendung eines individuellen Benutzerprofils, welches der Anwender vor Berechnung einer Route über ein Webportal pflegen muss.

Beide Ansätze dienen einem ersten Erkenntnisgewinn, inwieweit die Art der Routendarstellung überhaupt unterstützend für den Fahrer sein kann. Sofern das System ein solches Benutzerprofil automatisch generieren und pflegen soll, muss es die erreichten Wegpunkte sowie die befahrenen Streckenabschnitte registrieren und selbständig als "eher bekannt" oder "gut bekannt" kennzeichnen. Dazu bedarf es zusätzlicher Algorithmen, die die Bewertung bestimmter Streckenabschnitte automatisiert übernehmen können. Es ist geplant derartige Lernmecha- 
nismen zu integrieren, um die Interaktion mit dem System noch einfacher und weniger aufwändig für den Nutzer zu gestalten. Dabei soll stets gewahrt bleiben, dass der Nutzer die Kontrolle über den Prozess behält und die automatischen Prozesse auf Wunsch überschreiben bzw. deaktivieren kann.

\section{Literatur}

A. Al Mahmud, O. Mubin, and S. Shahid. User experience with in-car gps navigation systems: comparing the young and elderly drivers. In MobileHCl '09: Proceedings of the 11th International Conference on Human-Computer Interaction with Mobile Devices and Services, pages 1-2, New York, NY, USA, 2009. ACM.

M. Denis. The description of routes: A cognitive approach to the production of spatial discourse. Cahiers de Psychologie Cognitive, 16(4):409-458, 1997

J. Forlizzi, W. Barley, and T. Seder. Where should i turn: moving from individual to collaborative navigation strategies to inform the interaction design of future navigation systems. In Proceedings of the 28th international conference on Human factors in computing systems, pages 1261-1270. ACM, 2010.

M. Hipp, F. Schaub, F. Kargl, and M. Weber. Interaction Weaknesses of Personal Navigation Devices. 2010.

Institut für Demoskopie Allensbach. Innovationen treiben die Märkte, 2010.
A. Klippel, S. Hansen, K. Richter, and S. Winter. Urban granularities - a data structure for cognitively ergonomic route directions. Geolnformatica, 13(2):223-247, 2009.

A. Klippel, H. Tappe, and C. Habel. Pictorial representations of routes: Chunking route segments during comprehension. Spatial cognition III, pages 1034-1034, 2003.

K. Lovelace, M. Hegarty, and D. Montello. Elements of Good Route Directions in Familiar and Unfamiliar Environments. Spatial Information Theory. Cognitive and Computational Foundations of Geographic Information Science, 1661:751-751, 1999.

D. Münter, T. Hussein, and W. Gaulke. Kontextabhängige Empfehlung von Services zur intelligenten Navigationsunterstützung. In U. Schroeder, editor, Interaktive Kulturen - Proceedings der Workshops der Mensch \& Computer 2010 - 10. fachübergreifende Konferenz für interaktive und kooperative Medien, pages 138-142, 2010

K. Patel, M. Chen, I. Smith, and J. Landay. Personalizing Routes. In Proceedings of the 19th annual ACM symposium on User interface software and technology, pages 187-190. ACM, 2006

M. Pettitt, G. Burnett, and A. Stevens. Defining driver distraction. In Proc. World Congress on Intelligent Transport Systems, 2005.

K. Richter, M. Tomko, and S. Winter. A dialogdriven process of generating route directions. Computers, Environment and Urban Systems, 32(3):233-245, 2008.

M. Tomko. Destination descriptions in urban environments. PhD thesis, Citeseer, 2007.
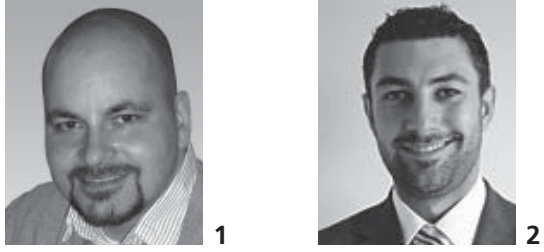

1 Daniel Münter ist wissenschaftlicher Mitarbeiter am Lehrstuhl für Interaktive Systeme der Universität Duisburg-Essen. Sein Forschungsinteresse gilt insbesondere der Entwicklung von Konzepten zur intelligenten Mobilitätsunterstützung. Darüber hinaus befasst er sich mit Fragestellungen zur Mensch-Service-Interaktion

2 Tim Hussein ist wissenschaftlicher Mitarbeiter am Lehrstuhl für Interaktive Systeme an der Universität Duisburg-Essen. Er leitet dort eine Forschergruppe, welche sich mit der Entwicklung intelligenter adaptiver Benutzerschnittstellen beschäftigt. Tim Hussein war und ist in verschiedenen geförderten Forschungsprojekten verantwortlich für Design, Implementierung und Evaluation adaptiver Systeme. 